\title{
Probing The Labelling and Isomorphic Graph of Generalized Fibonacci Caterpillar Tree
}

\author{
Bei-bei WANG ${ }^{1, a}$, Xin-sheng LIU ${ }^{1, b}$, Bing YAO ${ }^{1, c}$, Li-juan $\mathrm{QI}^{2, \mathrm{~d}}$ \\ ${ }^{1}$ College of Mathematics and Statistics, Northwest Normal University, Lanzhou, China \\ e-mail:924174362@163.com \\ 2Department of Basic Science of Lanzhou Polytechnical University, Lanzhou, China \\ Corresponding author e-mail:282455295@qq.com
}

\begin{abstract}
Keywords: generalized Fibonacci caterpillar tree; isomorphic tree; styling;set-ordered odd graceful labelling; odd-elegant labelling; super total graceful labelling; sun-tree; felicitous graph.
\end{abstract}

\begin{abstract}
All trees are graceful" has not been proven. Applying the construction method to show every generalized Fibonacci caterpillar tree is a set-ordered odd graceful graph meanwhile every generalized Fibonacci caterpillar tree not only have an odd-elegant labelling but also possess a super total graceful labelling. On this basis, we prove that the isomorphic graph of generalized Fibonacci caterpillar tree is a set-ordered graceful graph as well as a super total graceful graph. Finally, we show each generalized Fibonacci caterpillar sun-tree is a felicitous graph.
\end{abstract}

\section{Introduction}

As everyone know, graph labelling theory plays a important role in coding theory, complex networks, logistics and other fields. In operations research or systems theory and practice of systems enginnering. By applying graph labellings, one can divide large systems into subsystems. One, very often, utilize graph labellings to distinguish vertices and edges between vertices for the sake of searching certain fast algorithms to simulate efficient transmissions and communications in information spreading networks. There is a large and growing literature on graph labeling [1]. Rosa [2] testified if $G$ is graceful as well as overall its vertices are even defrees, and $|E(G) \equiv 0|$ or $3(\bmod 4)$. Cycle $C_{n}$ are graceful if and only if $n \equiv 0$ or $3(\bmod 4)$; paths $P_{n}$; wheels $W_{n}$ and complete bipartite graphs $K_{m, n}$ are graceful whereas complete graphs $K_{n}$ are graceful if and only if $n \leq 4$. It has been conjectured that all trees are graceful, it is still an open problem. Rosa contributed various labeling for solving problems of graph decementation and proposed some entertaining open questions. In 1979, Bermond [3] made conjecture: all lobster trees are graceful graphs, this conjecture has not been proven or negated. Sin-Min Lee [4] and others in 1991 proposed a conjecture "Every tree is a felicitous tree". This conjecture has the equal theoretical worth to "Every tree is a graceful tree" and also has the same difficulty to be proved. Both of them are NP-problems that have drew many researchers to be proved [5-9]. Many scholars attacked mathematical conjectures and made enormons contribution on graph theory. Theseworks prompt the graph labelling theory fleetly become a dynamic branch of graph labellings. In other words, graph labelling theory has played a important role in many fields.

In this paper the method of constructing the two graceful labelling proved that a new class of generalized Fibonacci caterpillar tree and its isomorphic graph is a set-ordered graceful graph, on this basis and prove that they are also super total graceful graph, then prove the tree $T$ stand for the generalized Fibonacci caterpillar isomorphic graph, if $T$ is consist of sun-trees we say that $T$ is a felicitous graph and it is short of GFCST graph.

We employ criterion symbol and technology of graph theory. Graphs make reference to loopless, no multiple edges, undirected, linked and limited, in addition to elsewise specified. A $(p, q)$-graph $G$ is one with $p$ vertices and $q$ edges. The steno symbol $[m, n]$ indicates an integer set $\{m, m+1, \ldots, n\}$, where $m$ and $n$ both are integers with $0 \leq m<n$; the mark $[s, t]^{\circ}$ figures an odd-set $\{s, s+1, \ldots, t\}$, where $s$ and $t$ both are odd integers with $1 \leq s<t$; and the mark $[k, l] l$ figures an even-set $\{k, k+2, \ldots, l\}$, where integers $k$ and $l$ both are even with $0 \leq k<l$.

Definition 1. ${ }^{[10,11]}$ The graph $G$ has $p$ vertices and q edges, then we grants a mapping $f: V(G) \rightarrow[0$, $2 q-1]$, and for distinct $v, u \in V(G)$ which satisfied $f(u) \neq f(v)$; About for every edges $v u$ of $G$, there 
correspond label $f(v u)$ satisfied $f(v u)=|f(v)-f(u)|$, and we can get that $f(v u)=[1,2 q-1]^{\circ}$ for every $v u \in E(G)$. So we name $f$ is an odd-graceful labeling as well as the graph $G$ is known as odd-graceful.

Definition 2. ${ }^{[12]}$ The $G$ has $p$ vertices and $q$ edges and is a bipartite graph. $\left(V_{1}, V_{2}\right)$ is the two bipartition of $G$. Suppose $G$ exists an odd-graceful labeling $f$ which satisfieds $\max \{f(y) \mid y \in Y\}<\min \{f(x)$ $\mid x \in X\}$, then we say $f$ is a set-ordered odd-graceful labeling.

Definition 3. ${ }^{[13,14]}$ The graph $G$ has $p$ vertices and q edges, then we grants a mapping $f: V(G) \rightarrow[0$, $2 q-1]$, and for distinct $v, u \in V(G)$ which satisfied $f(u) \neq f(v)$; but for every $u v \in E(G)$, there correspond label $f(v u)$ satisfied $f(v u)=f(v)+f(u)(\bmod 2 q)$ as well as $f(v u)=[1,2 q-1]^{\circ}$, so we say $f$ is an odd-elegant labeling, $G$ is knnown as odd-elegant graph.

Definition 4. ${ }^{[15,16]}$ A total graceful labeling (TGL) of a $(p, q)$ graph $G$ is a bijection $f$ from $V(G) \cup E(G)$ to the set $\{1,2,3, \ldots, p+q\}$ so that $f(u v)=|f(u)-f(v)|$ for all $u v \in E(G)$. A $T G L$ is called a super TGL if $f(E)=\{1,2,3, \ldots, q\}$. A graph that admits a super total graceful labeling is called a super total graceful graph (STGG).

Definition 5. ${ }^{[6]}$ The graph $\mathrm{G}$ has $\mathrm{p}$ vertices and q edges, then we grants a mapping $f: V(G) \rightarrow[0, q]$, and for distinct $v, u \in V(G)$ which satisfied $f(v) \neq f(u)$. A felicitous labelling called $f$ of graph $G$ satisfied that for distinct $v, u \in V(G), f(v) \neq f(u)$, and foe every edge $v u \in E(G)$, there have $f(v u)=f(v)+f(u)(\bmod q)$ and $f(u)=[0, q-1]$. Then we call $G$ is a felicitous graph.

Definition 6. The $\mathrm{G}$ has $\mathrm{p}$ vertices and $\mathrm{q}$ edges and is a bipartite graph. $\left(V_{1}, V_{2}\right)$ is the two bipartition of $G$. Suppose $G$ exists a felicitous labelling $f$ which satisfieds $\max \{f(y) \mid y \in Y\}<\min \{f(x) \mid x \in X\}$, now we named $f$ is a set-ordered felicitous labelling, $G$ is knnown as a set-ordered felicitous graph, and write this case as $f(X)<f(Y)$.

For a path $p=a_{0} a_{1} a_{2}, \ldots, a_{n}$, we add new vertices $a_{i, 1}, a_{i, 2}, \ldots, a_{i, m_{i}}$ to each vertex $a_{i}$ by edges $a_{i} a_{i j}$ with $i=1,2, \ldots, n$ and $j=1,2, \ldots, m_{i}\left(m_{i} \geq 0\right)$, call such tree a caterpillar tree. For a caterpillar tree $T$, if $m_{1}=a, m_{2}=b(a \geq b \geq 1)$ and $m_{i}=m_{i}-2+m_{i}-1$ for $i \geq 2$ hold true, we call $T$ a generalized Fibonacci caterpillar tree, and denoted by $F_{n}, a_{0}$ is called the $s$-head of $F_{n}$ as well as $a_{n, m_{n}}$ the $L$-head of $F_{n}$.

Let $T_{i}$ is a copy of $q$ generalized Fibonacci caterpillar tree $F_{n}, i=1,2, \ldots, \beta$. Join the $L$-head $a_{n, m_{n}}^{i}$ of the tree $T_{i}$ with the $s$-head $a_{0}^{i+1}$ of the tree $T_{i+1}$ by an edge $(i=1,2, \ldots, \beta-1)$, and joining the $L$-head of the tree $T_{\beta}$ with the s-head of the tree $T_{1}$ with an edge. And the resultant tree is named a generalized Fibonacci caterpillar sun-tree (GFCST).

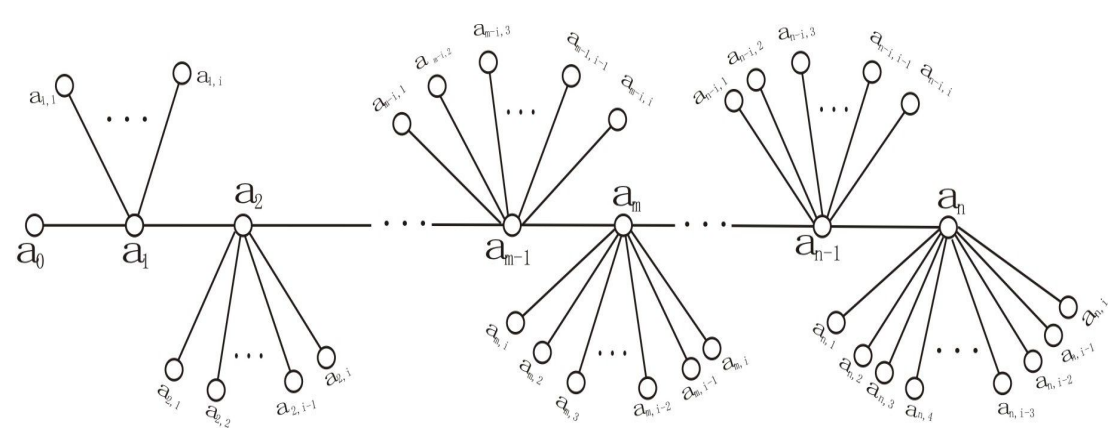

Fig 1. Generalized Fibonacici caterpillar tree.

\section{Main Results}

Theorem 1. Every generalized Fibonacci caterpillar tree $F_{n}$ is a set-ordered odd graceful tree.

Proof. Let $F_{n}$ be a generalized Fibonacci caterpillar tree defined Section 1. We label the vertices of each generalized Fibonacci's caterpillar graphs via a labelling $f$ established in the following:

$f\left(a_{n}\right)=0 ; f\left(a_{n, n}\right)=2 q-1, f\left(a_{n, j}\right)=2 q-1-2\left(m_{n}-j\right), j \in\left\{1,2, \ldots, m_{n}\right\}$;

$f\left(a_{n-1}\right)=f\left(a_{n, 1}\right)-2, f\left(a_{n-1, j}\right)=f\left(a_{n}\right)+2\left(m_{n-1}-j+1\right), j \in\left\{1,2, \ldots, m_{n-1}\right\}$;

$f\left(a_{n-2}\right)=f\left(a_{n-1,1}\right)+2, f\left(a_{n-2, j}\right)=f\left(a_{n-1}\right)-2\left(m_{n-2}-j+1\right), j \in\left\{1,2, \ldots, m_{n-2}\right\}$;

$f\left(a_{n-3}\right)=f\left(a_{n-2,1}\right)-2, f\left(a_{n-3, j}\right)=f\left(a_{n-2}\right)+2\left(m_{n-3}-j+1\right), j \in\left\{1,2, \ldots, m_{n-3}\right\}$; 
$f\left(a_{n-4}\right)=f\left(a_{n-3,1}\right)+2, f\left(a_{n-4, j}\right)=f\left(a_{n-3}\right)-2\left(m_{n-4}-j+1\right), j \in\left\{1,2, \ldots, m_{n-4}\right\}$

$f\left(a_{n-5}\right)=f\left(a_{n-4,1}\right)-2, f\left(a_{n-5, j}\right)=f\left(a_{n-4}\right)+2\left(m_{n-5}-j+1\right), j \in\left\{1,2, \ldots, m_{n-5}\right\}$;

$f\left(a_{n-6}\right)=f\left(a_{n-5,1}\right)+2, f\left(a_{n-6, j}\right)=f\left(a_{n-5}\right)-2\left(m_{n-6}-j+1\right), j \in\left\{1,2, \ldots, m_{n-6}\right\}$;

In general, on the basis of the above, we can easily find the following equation:

$$
\begin{aligned}
& f\left(a_{k}\right)=f\left(a_{k+1,1}\right)-2, f\left(a_{k, j}\right)=f\left(a_{k+1}\right)+2\left(m_{k}-j+1\right), j \in\left\{1,2, \ldots, m_{k}\right\} ; \\
& f\left(a_{k-1}\right)=f\left(a_{k, 1}\right)+2, f\left(a_{k-1, j}\right)=f\left(a_{k}\right)-2\left(m_{k-1}-j+1\right), j \in\left\{1,2, \ldots, m_{k-1}\right\} .
\end{aligned}
$$

And then we also find that equation: $f\left(a_{k}\right)=f\left(a_{k+1,1}\right)-2, f\left(a_{k, j}\right)=f\left(a_{k+1}\right)+2\left(m_{k}-j+1\right), j \in\left\{1,2, \ldots, m_{k}\right\}$

to show vertices which labeling are odd numbers, so we can note the set make up by this vertices is $Y$, as well as the equation: $f\left(a_{k-1}\right)=f\left(a_{k, 1}\right)+2, f\left(a_{k-1, j}\right)=f\left(a_{k}\right)-2\left(m_{k-1}-j+1\right), j \in\left\{1,2, \ldots, m_{k-1}\right\}$

to show vertices which labeling are even numbers, so we can note the set make up by this vertices is $X$.

So that, we can see $f\left(a_{n}\right)=0, f\left(a_{0} a_{1}\right)=0$ and $f\left(a_{n, n}\right)=2 q-1$. Clearly, $f\left(x_{i}\right)$ is even $i \in[1, s]$; and $f\left(y_{j}\right)$ is odd $j \in[1, t-1]$, and $\max \{f(x) \mid x \in X\}<\min \{f(y) \mid y \in Y\} . f\left(x_{i+1}\right)<f\left(x_{i}\right)$ for $i \in[1, s] ; f\left(x_{j+1}\right)<f\left(x_{j}\right)$ for $j \in[1$, $t-1]$, and The certification is completed.

Theorem 2. If generalized Fibonacci caterpillar tree $F_{n}$ is set-ordered odd-graceful graph, then it is odd-elegant graph.

Proof. According to Theorem 1, we know every generalized Fibonacci caterpillar tree $F_{n}$ exsits a set-ordered odd-graceful labelling $f$. Now we regulate one new labelling $h$ of $F_{n}$ as below:

$h\left(a_{n}\right)=f\left(a_{n}\right)=0 ; \quad h\left(a_{n, n}\right)=2 q-f\left(a_{n, n}\right)=1, h\left(a_{n, j}\right)=2\left(m_{n}-j\right)+1, j \in\left\{1,2, \ldots, m_{n}-1\right\} ;$

$h\left(a_{n-1}\right)=2(q+1)-f\left(a_{n, 1}\right), h\left(a_{n-1, j}\right)=f\left(a_{n-1, j}\right), j \in\left\{1,2, \ldots, m_{n-1}\right\}$;

$h\left(a_{n-2}\right)=f\left(a_{n-2}\right), h\left(a_{n-2, j}\right)=2 q+2\left(m_{n-2}-j+1\right)-f\left(a_{n-1}\right), j \in\left\{1,2, \ldots, m_{n-2}\right\}$;

$h\left(a_{n-3}\right)=2(q+1)-f\left(a_{n-2,1}\right), h\left(a_{n-3, j}\right)=f\left(a_{n-3, j}\right), j \in\left\{1,2, \ldots, m_{n-3}\right\}$;

$h\left(a_{n-4}\right)=f\left(a_{n-4}\right), h\left(a_{n-4, j}\right)=2 q+2\left(m_{n-4}-j+1\right)-f\left(a_{n-3}\right), j \in\left\{1,2, \ldots, m_{n-4}\right\}$;

$h\left(a_{n-5}\right)=2(q+1)-f\left(a_{n-4,1}\right), h\left(a_{n-5, j}\right)=f\left(a_{n-5, j}\right), j \in\left\{1,2, \ldots, m_{n-5}\right\}$;

$h\left(a_{n-6}\right)=f\left(a_{n-6}\right), h\left(a_{n-6, j}\right)=2 q+2\left(m_{n-6}-j+1\right)-f\left(a_{n-6}\right), j \in\left\{1,2, \ldots, m_{n-6}\right\}$.

Above all, we can promote formula to general case:

$h\left(a_{k}\right)=f\left(a_{k}\right), h\left(a_{k, j}\right)=2 q+2\left(m_{k}-j+1\right)-f\left(a_{n+1}\right), j \in\left\{1,2, \ldots, m_{k}\right\}$;

$h\left(a_{k+1}\right)=2(q+1)-f\left(a_{k+2,1}\right), h\left(a_{k+1, j}\right)=f\left(a_{k+1, j}\right), j \in\left\{1,2, \ldots, m_{k-1}\right\}$.

Furthermore,

$h\left(a_{k}\right)=f\left(a_{k}\right), h\left(a_{k+1, j}\right)=f\left(a_{k+1, j}\right), j \in\left\{1,2, \ldots, m_{k-1}\right\}$ is even. $h\left(a_{k, j}\right)=2 q+2\left(m_{k}-j+1\right)-f\left(a_{n+1}\right), j \in\left\{1,2, \ldots, m_{k}\right\}$, $h\left(a_{k+1}\right)=2(q+1)-f\left(a_{k+2,1}\right)$ is odd. And $h\left(E\left(F_{n}\right)\right)=2 q-f\left(E\left(F_{n}\right)\right), f\left(E\left(F_{n}\right)\right)=[1,2 q-1]^{\circ}$, then we have: $h\left(E\left(F_{n}\right)\right)=[1,2 q-1]^{\circ}$. In consequence, $h$ is an odd-elegant labelling of $F_{n}$. (See figure 2)

Theorem 3. Every generalized Fibonacci caterpillar tree $F_{n}$ is the super total graceful tree.

Proof. According to Theorem 1, let $f$ be a set-ordered graceful labeling of the graph. Then we define a new labeling $g$, such that:

$g\left(a_{n}\right)=p ; g\left(a_{n, n}\right)=2 q-1+p, g\left(a_{n, j}\right)=2 q-1-2\left(m_{n}-j\right)+p, j \in\left\{1,2, \ldots, m_{n}\right\}$;

$g\left(a_{n-1}\right)=g\left(a_{n, 1}\right)-2+p, g\left(a_{n-1, j}\right)=g\left(a_{n}\right)+2\left(m_{n-1}-j+1\right)+p, j \in\left\{1,2, \ldots, m_{n-1}\right\}$;

$g\left(a_{n-2}\right)=g\left(a_{n-1,1}\right)+2+p, g\left(a_{n-2, j}\right)=g\left(a_{n-1}\right)-2\left(m_{n-2}-j+1\right)+p, j \in\left\{1,2, \ldots, m_{n-2}\right\}$;

$g\left(a_{n-3}\right)=g\left(a_{n-2,1}\right)-2+p, g\left(a_{n-3, j}\right)=g\left(a_{n-2}\right)+2\left(m_{n-3}-j+1\right)+p, j \in\left\{1,2, \ldots, m_{n-3}\right\}$;

$g\left(a_{n-4}\right)=g\left(a_{n-3,1}\right)+2+p, g\left(a_{n-4, j}\right)=g\left(a_{n-3}\right)-2\left(m_{n-3}-j+1\right)+p, j \in\left\{1,2, \ldots, m_{n-4}\right\}$;

...

$g\left(a_{k}\right)=g\left(a_{k+1,1}\right)-2+p, g\left(a_{k, j}\right)=g\left(a_{n+1}\right)+2\left(m_{k}-j+1\right)+p, j \in\left\{1,2, \ldots, m_{k}\right\}$

Hence, $g$ is a super total graceful labeling of $F_{n}$. The theorem is covered.

Corollary 1. Every generalized Fibonacci caterpillar isomorphic graph is a set-ordered odd graceful graph also a super total graceful graph.

Theorem 4. Every generalized Fibonacci caterpillar sun-tree (GFCST) is a felicitous graph.

Proof. According the definition 4, let $T_{i}$ is a GFCST. Let $X_{i}, Y_{j}$ be the bipartition of a bipartite graph $G$, so that $V\left(T_{i}\right)=X_{i} \cup Y_{j}$, where $X_{i}=\left\{x_{i} \in[1, s]\right\}$ and $Y_{j}=\left\{y_{j} \in[1, t]\right\}$ with $s+t=\left|T_{i}\right|$. Every $T_{i}$ acknowledges a set-ordered felicitous labelling $f$ such that: $\max \left\{f\left(x_{i}\right) \mid x \in X_{i}\right\}<\min \left\{f\left(y_{j}\right) \mid y \in Y_{j}\right\}$. Without loss of generality, we have $f\left(x_{k}\right)=k-1, k \in[1, s]$ and $f\left(y_{j}\right)=j+s-1, j \in[1, t]$ when $i \in[1, n]$, join in an edge between vertex $X_{1}^{i}$ and $Y_{1}^{i+1}$, and connect $X_{s}^{n}$ and $X_{1}^{1}$ with an edge, then we can obtain a GFCST in Theorem 3. Defined a new labelling $g$ for GFCST as follow:

$$
g\left(x_{k}^{i}\right)=\frac{1}{2}(s+t)(i-1)+k-1, i \in[1, n]^{o}, k \in[1, s] ; g\left(y_{j}^{i}\right)=\frac{1}{2}(s+t)(n+i)+\frac{1}{2} t(i-2)+j-1, i \in[1, n]^{l}, j \in[1, t] \text {; }
$$




$$
\begin{aligned}
& g\left(x_{k}^{i}\right)=\frac{1}{2} s(n+1)+\frac{1}{2} i(s+t)+1 / 2 t(n-1)-k, i \in[1, n]^{o}, k \in[1, s] ; g\left(y_{j}^{i}\right)=\frac{1}{2} i(s+t)-r, i \in[1, n]^{l}, j \in[1, t] ; \\
& g\left(x_{k}^{i} y_{j}^{i}\right)=g\left(x_{k}^{i}\right)+g\left(y_{j}^{i}\right), i \in[1, n]^{o}, k \in[1, s], j \in[1, t] ;
\end{aligned}
$$

now, it is easily testify the set of vertex labelling satisfies

$$
\left\{g\left(x_{k}^{i}\right) \mid i \in[1, n]^{o}, k \in[1, s]\right\} \cup\left\{g\left(y_{j}^{i}\right) \mid i \in[1, n]^{l}, j \in[1, t]\right\}=\left[0, \frac{1}{2}(s+t)(n-1)+s-1\right],
$$

and

$$
\left\{g\left(y_{j}^{i}\right) \mid i \in[1, n]^{o}, j \in[1, t]\right\} \cup\left\{g\left(x_{k}^{i}\right) \mid i \in[1, n]^{l}, k \in[1, s]\right\}=\left[\frac{1}{2}(s+t)(n-1)+s, n(s+t)-1\right]
$$

we notice that the total vertex is not variable in the process of constructing GFCST. Furthermore the number of edge of GFCST is $\mid(E(G) \mid=n(s+t)$. So we have

and

$$
g(V(G))=[0, n(s+t)-1] \subseteq[0, n(s+t)]
$$

$g\left(E\left(T_{1} \cup T_{2} \cup \ldots \cup T_{n}\right)=\left[\frac{1}{2}(s+t)(n-1)+s-1, \frac{1}{2}(s+t)(3 n-1)+s-2\right] \subseteq[0, n(s+t)-1]\right.$

Judging by the Definition 4, the labelling of $g$ is a felicitous labelling of GFCST. In other words, the GFCST is the felicitous graph. (See figure 3)

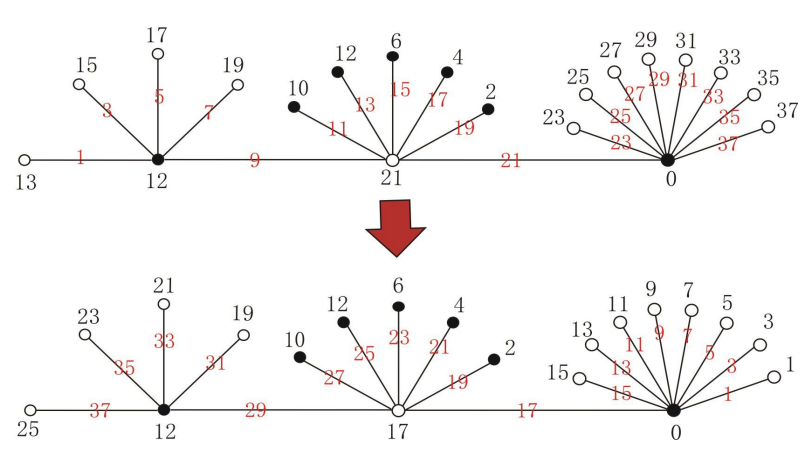

Fig 2. An odd-elegant of generalized Fibonacci caterpillar tree $F_{3}$.

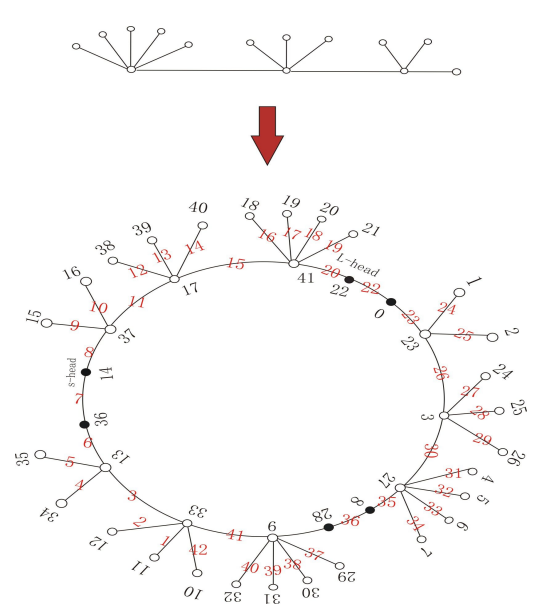

Fig 3. A felicitous labelling of GFCST.

\section{Conclusions}

Cleary, the results, here, are suitable for constructing graceful trees or bipartite graceful trees. Most of proofs of the results in this note are effective. We have defined a new labelling called generalized Fibonacci caterpillar tree labelling, in order to study more complex models from observation and analysis of graphs. First, we validate the generalized Fibonacci caterpillar tree not only is set-ordered odd graceful tree but also is an odd-elegant garph meanwhile is super total graceful tree. Besides, we give a proof for illustrating set-ordered odd graceful labellings as well as odd-elegant labelling and super total graceful labelling. Finally, we provide the method for stating the isomorphic graph of generalized Fibonacci caterpillar tree labelling sun-tree is a felicitous graph. It could be available to indicate more complicated grapg labelling that acknowledge graceful labellings and GFCST-labellings, and to discover some underlying applications of these labellings. As a byproduct it is easy to distinguish new classes of graceful graphs by using graceful similar systems built upon known smaller graceful trees, which are gracefully similar. It could be interesting to probe and identify more related concepts and relationships among them. For example, it could be nice to try to work out the specific conditions when the graph is a strongly graceful.

At the end of this article, we propose several problems for further research as follows

Problem 1. Adding leaves to the set-ordered odd graceful generalized Fibonacci caterpillar trees, could we produce the tree is set-ordered odd graceful and an odd-elegant graph ? 
Problem 2. Could we can use the odd-elegant labelling $f$ of generalized Fibonacci caterpillar trees produce a strong set-ordered odd graceful?

Problem 3. Whether all higher-order Fibonacci caterpillar trees and their isomorphic graphs are set-ordered odd graceful, also, super total graceful ?

Problem 4. If every Fibonacci caterpillar trees exist a super total magic labeling, furthermore, or every Fibonacci caterpillar trees admits a super $(k, \lambda)$-magically total labelling for some integers $k$ and $\lambda \neq 0$ ?

\section{Acknowledgment}

This research was supported by the National Natural Science Foundation of China under grant No. 61163054, No. 61363060 and No. 61163037.

\section{References}

[1] J.A. Gallian, A dynamic survey of graph labeling, Electron.J. Combin. 13(2010) .

[2] Rosa A. On certain valuations of the vertices of a graph, Theory of Graphs, International Symposium, Rome, July 1966. New York: Gordon and Breach, 1967,349-355.

[3] BERMOND J C. Graceful Graphs, Radio Antennae and French Windmills. London:Graph Theory and Comhinatorics Pitman,1979,18-37.

[4]S-Min Lee, E. Schmeichel, and S.C.Shee. On felicitous sun graphs.Discrete Math.,1991,93,201-209.

[5]X-S-Liu, Y-Y-Liu, B-Yao, Y-Gou. Type of odd-graceful labelings of some dragon graphs. Journal of Lanzhou University(Natural Sciences), 2014, 50(4),541-545.

[6]M-K, M-M, kala R. Some results on felicitous labelling of graphs. J Combin Math Combin Comput, 2012,81:273.

[7]Samina Abbas Boxwala, Priyam Vashishta. Some new on families of Graceful Graphs. Electronic Notes in Discrete Mathematics, 48(2015),127-133.

[8]X-Q-Zhou, B-Yao, X-E-Chen. Every Lobster Is Odd-elegant. Information Processing Letters 113(2013),30-33.

[9]X-Q-Zhou, B-Yao, X-E-Chen and H-X-Tao. A proof to the odd-gracefulness of all lobsters. Ars Combinatoria 103(2012),13-18.

[10] B-Yao, H-Cheng, M-Yao, et al. A Note on Graceful Trees.Ars Combinatoria, 2009, 92: 155-161.

[11]R.B. Gnanajothi, Topics in graph theory, PhD thesis, Madurai Kamaraj University, 1991.

[12]B-Yao, H-Cheng, M-Yao, et al. A note on strongly graceful trees, Ars Combin.2009, 92,155-169.

[13]X-Q-Zhou, B-Yao, X-E-Chen. Every lobster is odd-elegant, Information Processing Letters, 113 (2013) 30-33.

[14]X-Q-Zhou, The research on graceful odd-graceful and odd-elegant labelling of graphs[D]: [master graduate paper], Northwest Normal University, 2013.

[15]J.-G. Wang, D.-J. Jin, X.-G. Lu, D. Zhang, The gracefulness of a class of lobster trees, Math. Comput. Modelling, 1994, 20 (9),105-110.

[16] S.P.Subbiah, J. Pandimadevi, R.Chithra, Super total graceful graphs,Electronic Notes in Discrete Mathematics. 2015, 48,301-304. 